\title{
Systematic review of charged-particle therapy for chordomas and sarcomas of the mobile spine and sacrum
}

\author{
Zach Pennington, BS, ${ }^{1}$ Jeff Ehresman, BS, ${ }^{1}$ Aladine A. Elsamadicy, MD, ${ }^{2}$ John H. Shin, MD, ${ }^{3}$ \\ C. Rory Goodwin, MD, PhD, ${ }^{4}$ Joseph H. Schwab, MD, ${ }^{5}$ and Daniel M. Sciubba, MD ${ }^{1}$ \\ 1Department of Neurosurgery, Johns Hopkins University School of Medicine, Baltimore, Maryland; 'Department of Neurosurgery, \\ Yale University School of Medicine, New Haven, Connecticut; ${ }^{3}$ Department of Neurosurgery, Massachusetts General Hospital, \\ Harvard Medical School, Boston, Massachusetts; ${ }^{2}$ Department of Neurosurgery, Duke University Medical Center, Durham, \\ North Carolina; and ${ }^{5}$ Department of Orthopaedic Surgery, Massachusetts General Hospital, Harvard Medical School, Boston, \\ Massachusetts
}

OBJECTIVE Long-term local control in patients with primary chordoma and sarcoma of the spine and sacrum is increasingly reliant upon en bloc resection with negative margins. At many institutions, adjuvant radiation is recommended; definitive radiation is also recommended for the treatment of unresectable tumors. Because of the high off-target radiation toxicities associated with conventional radiotherapy, there has been growing interest in the use of proton and heavyion therapies. The aim of this study was to systematically review the literature regarding these therapies.

METHODS The PubMed, OVID, Embase, and Web of Science databases were queried for articles describing the use of proton, combined proton/photon, or heavy-ion therapies for adjuvant or definitive radiotherapy in patients with primary sarcoma or chordoma of the mobile spine and sacrum. A qualitative synthesis of the results was performed, focusing on overall survival (OS), progression-free survival (PFS), disease-free survival (DFS), and disease-specific survival (DSS); local control; and postradiation toxicities.

RESULTS Of 595 unique articles, 64 underwent full-text screening and 38 were included in the final synthesis. All studies were level III or IV evidence with a high risk of bias; there was also significant overlap in the reported populations, with six centers accounting for roughly three-fourths of all reports. Five-year therapy outcomes were as follows: proton-only therapies, OS $67 \%-82 \%$, PFS $31 \%-57 \%$, and DFS $52 \%-62 \%$; metastases occurred in $17 \%-18 \%$ and acute toxicities in 3\%-100\% of cases; combined proton/photon therapy, local control $62 \%-85 \%$, OS $78 \%-87 \%$, PFS $90 \%$, and DFS $61 \%-72 \%$; metastases occurred in 12\%-14\% and acute toxicities in $84 \%-100 \%$ of cases; and carbon ion therapy, local control $53 \%-100 \%$, OS $52 \%-86 \%$, PFS (only reported for 3 years) $48 \%-76 \%$, and DFS $50 \%-53 \%$; metastases occurred in $2 \%-39 \%$ and acute toxicities in $26 \%-48 \%$. There were no studies directly comparing outcomes between photon and charged-particle therapies or comparing outcomes between radiation and surgical groups.

CONCLUSIONS The current evidence for charged-particle therapies in the management of sarcomas of the spine and sacrum is limited. Preliminary evidence suggests that with these therapies local control and OS at 5 years are comparable among various charged-particle options and may be similar between those treated with definitive charged-particle therapy and historical surgical cohorts. Further research directly comparing charged-particle and photon-based therapies is necessary.

https://thejns.org/doi/abs/10.3171/2021.2.FOCUS201059

KEYWORDS chordoma; carbon ion therapy; proton therapy; radiotherapy; local control; adjuvant

$\mathrm{E}$ $\mathrm{N}$ BLOC resection with negative margins (R0 resection) improves local control (LC) and disease-free survival (DFS) in patients with chordoma and primary sarcomas of the spinal column and sacrum. ${ }^{1-5} \mathrm{R} 0$ resection may also improve overall survival (OS)., ${ }^{1,6}$ Sur- gery has been the mainstay of therapy for years as these tumors are radioresistant and the doses required to improve LC were unacceptably toxic to local healthy tissues. Certain locations, such as the mobile spine, offer unique challenges given the often close approximation of tumor

ABBREVIATIONS CIRT = carbon ion radiotherapy; CTC = Common Toxicity Criteria; DFS = disease-free survival; DSS = disease-specific survival; LC = local control; LFU = last follow-up; MGH = Massachusetts General Hospital; OS = overall survival; PFS = progression-free survival; PRISMA = Preferred Reporting Items for Systematic Reviews and Meta-Analyses; RBE = relative biological effectiveness; RFS = relapse-free survival.

SUBMITTED December 14, 2020. ACCEPTED February 23, 2021

INCLUDE WHEN CITING DOI: 10.3171/2021.2.FOCUS201059. 
and eloquent nervous tissue. In the latter part of the 20th century, though, more advanced radiation modalities became clinically available as adjuvant or definitive therapy, including focused photon radiotherapy modalities and charged-particle therapies, notably proton therapy and carbon ion radiotherapy (CIRT). Charged particles have superior behaviors in soft tissue that theoretically enable them to provide more focused delivery of radiation energy to the target site with minimal off-target effects.

Preliminary studies, such as those from the Massachusetts General Hospital (MGH), ${ }^{7,8}$ found that the addition of modern radiation therapy could improve LC in patients operated on for spinal chordoma. Importantly, LC rates improved even in patients with positive surgical margins. More recent evidence has suggested that adjuvant radiation with these advanced modalities may be even more important to LC than surgical margins, ${ }^{9}$ perhaps due to the presence of micrometastatic disease located immediately outside the tumor pseudocapsule. As such, there is increased interest among spine surgeons, medical oncologists, and radiation oncologists in the use of proton therapy and hadron therapy as either definitive or adjuvant radiotherapy for patients with chordoma and sarcoma of the spinal column. In this study, we sought to systematically review the existing literature with a focus on radiation regimens, LC, DFS, progression-free survival (PFS), OS, and postradiotherapy complications. Our main objective was to compare the efficacy of proton, mixed proton/ photon, and carbon ion radiotherapy in terms of the above outcomes, focusing on LC, PFS, DFS, and OS at 5 years following radiation treatment. We included both surgeryand radiation-only series to address the second question of whether definitive radiotherapy with charged particles can produce 5-year LC, OS, DFS, and PFS rates similar to the rates reported in historical surgical series.

\section{Methods}

A literature search was conducted on November 13, 2020, to identify all published reports of proton therapy, combined proton therapy/photon tomotherapy, and hadron therapy for patients with primary chordoma or sarcoma of the mobile spine and sacrum. Queried databases included PubMed/Medline, Embase, OVID Medline, and Web of Science. We also queried the bibliographies of included articles to identify additional articles. The search query for the PubMed/Medline database was ("chordoma" OR "chondrosarcoma" OR "osteosarcoma" OR "osteogenic sarcoma" OR "Ewing sarcoma" OR "Ewing's sarcoma" OR "Ewings sarcoma" OR "primary bone tumor" OR "primary vertebral tumor" OR "primary spine tumor") AND (spine OR spinal OR vertebral OR vertebra OR vertebrae OR sacrum OR sacral) AND ("carbon ion" OR "hadron" OR "proton" OR "proton therapy" OR "carbon ion therapy" OR "hadron therapy" OR "charged ion" OR "charged ion therapy"). The individual queries for each database are listed in the Appendix.

Included studies had full-text English translations and included a minimum of 5 adult patients ( $>16$ years of age) being treated for a primary sarcoma or chordoma of the mobile spine or sacrum. Articles had to provide pri- mary data on one of the following outcomes of interest: OS, PFS, disease-specific survival (DSS), DFS, LC, rates of metastasis, and radiation-associated toxicities. Articles were excluded if they pooled adult and pediatric patients, pooled spine/sacral lesions with lesions of other sites, pooled patients treated with pure photon regimens with those receiving proton/charged-particle regimens, or presented nonprimary data (i.e., fit one of the following article types: commentary, opinion, perspective, systematic review, narrative review). Articles were screened by two reviewers (Z.P. and J.E.), with a third reviewer (D.M.S.) serving as referee in case of disagreement. Screening was performed using Covidence v2313 (Covidence) according to the Preferred Reporting Items for Systematic Reviews and Meta-Analyses (PRISMA) statement. ${ }^{10}$

We collected details about patient demographics (age, sex, median tumor clinical target volume), radiation treatment modality (protons, mixed protons and photons, CIRT, or other hadron therapy), dosing schema (total dose, fractionation, duration), and concomitant treatments given with radiation (including surgery, chemotherapy, and hyperthermia). All studies were case series and were deemed to have a high risk of bias.

\section{Results}

In total, 595 unique articles were identified, of which 64 underwent full-text screening and 36 were included in the final qualitative synthesis (Fig. 1). ${ }^{1,11-45}$ The most common exclusion reasons were pooling of spine/sacrum lesions with other lesion locations $(n=14)$, the use of photon-only radiotherapy $(n=9)$, and failure to report one of the outcomes of interest $(\mathrm{n}=3)$. Of the included articles, 8 examined proton-only therapy (Table 1), ${ }^{11,22,33,40-44} 11$ examined combined proton/photon radiotherapy (Table 2), ${ }^{1,12-20,45}$ 14 examined CIRT (Table 3), ${ }^{21,23-32,34-36}$ and 3 examined a combination of proton radiotherapy and CIRT or another hadron therapy (Table 4). ${ }^{37-39}$ All included studies were at high risk of reporting bias. All were level IV evidence based on the North American Spine Society Levels of Evidence, ${ }^{46}$ except the study by Mima et al. ${ }^{37}$ (level III evidence). Most studies were published by five centers: the Heidelberg Ion Beam Therapy Center $(\mathrm{n}=3),{ }^{21,31,34}$ the Hyogo Ion Beam Medical Center in Japan $(n=4),{ }^{11,23,37,39}$ the MGH $(n=9), 1,13-17,19,20,22$ the National Institute of Radiological Sciences in Chiba, Japan $(\mathrm{n}=10),{ }^{23-29,32,33,36}$ and the Paul Scherrer Institute $(n=4) .{ }^{41-44}$ Unless otherwise stated, the results are reflective of adjuvant particle radiation before, after, or combined with surgery.

\section{Proton-Only Series}

All 8 studies ${ }^{11,22,33,40-44}$ were small (5-116 patients). The median age was 50-71 years, the median follow-up was 18-65 months, and patients were 55\%-100\% male. Excluding the study by Murray et al. ${ }^{41}$ which separated chordoma and chondrosarcoma outcomes, the most common pathologies were chordoma (67\%-100\% of patients) and chondrosarcoma $(0 \%-33 \%)$. Five series ${ }^{11,22,42-44}$ described outcomes for only chordoma patients, and $0 \%-75 \%$ of lesions involved the mobile spine; 2 reported only outcomes for sacral chordoma. ${ }^{11,44} \mathrm{LC}$ was $56 \%-68 \%$ at 5 


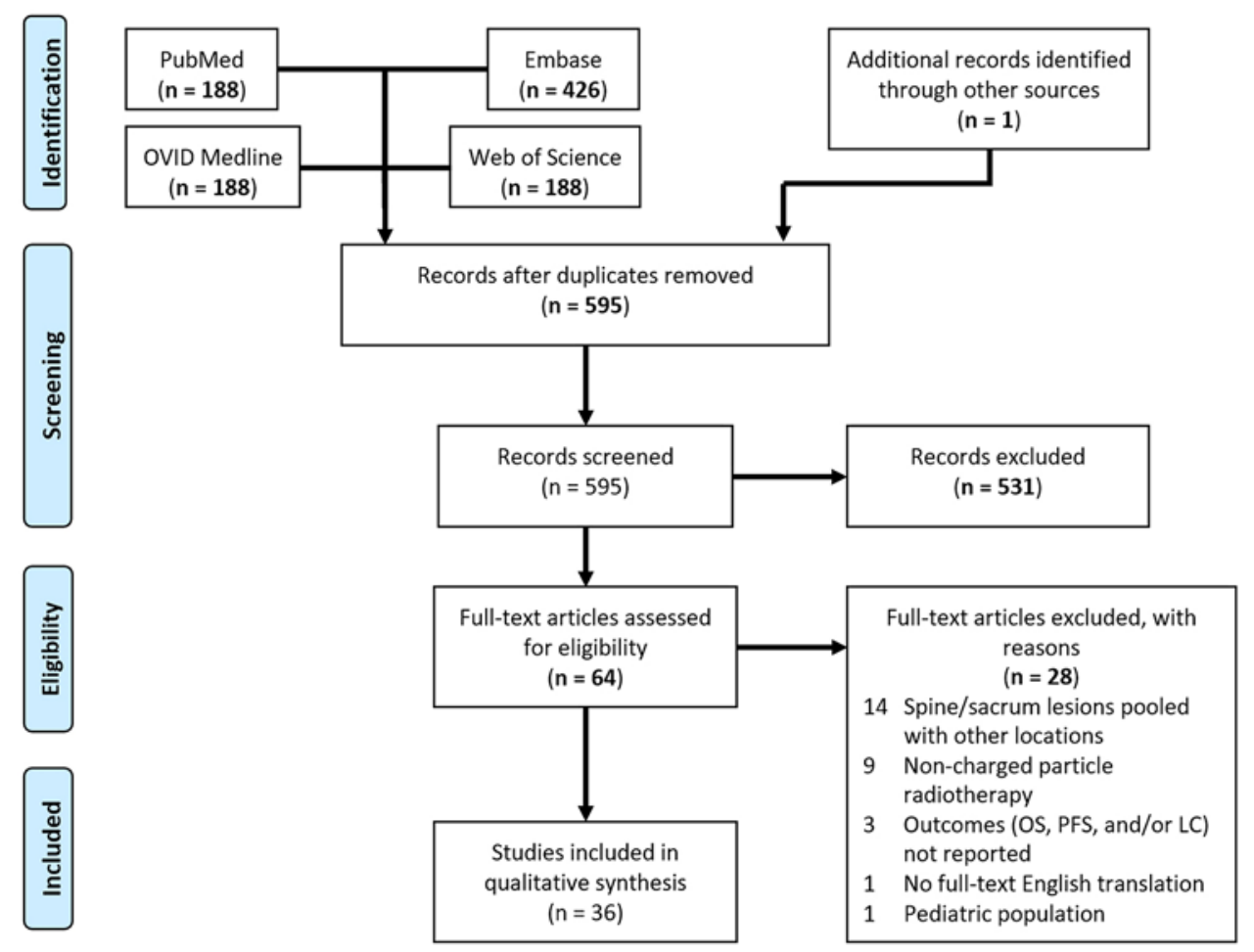

FIG. 1. PRISMA flow diagram for study queries.

years and $62 \%-100 \%$ at the last follow-up (LFU). Fiveyear outcomes were OS $67 \%-82 \%$, PFS $31 \%-57 \%$, and DFS $52 \%-62 \%$. Metastases occurred in $17 \%-18 \%$ by the LFU. Acute toxicities were seen in 3\%-100\% of patients with National Cancer Institute Common Toxicity Criteria (CTC) events $\geq$ grade 3 noted in $0 \%-20 \%$. Late toxicities occurred in $34 \%-60 \%$, with $0 \%-16 \%$ suffering toxicities $\geq$ grade 3 . The median dose relative biological effectiveness (RBE) ranged from 70 to 74 ; most used fractions of 1.8-2.2 RBE. No common outcomes were reported by the 2 studies of nonsurgical patients. ${ }^{11,44}$ However, Aibe et al. ${ }^{11}$ reported the following 3-year outcomes: LC $82 \%$, OS $93 \%$, PFS $90 \%$, and DFS $81.9 \%$.

\section{Mixed Proton/Photon Series}

Eleven studies described combined proton/photon therapy. ${ }^{1,12-20,45}$ All series were small (11-126 patients) with a median age of 39-70 years, sex makeup of 45\%-63\% male, and median follow-up of 12.9-99.6 months; $54 \%-100 \%$ of patients were treated for chordoma with 6 series $^{1,12-14,19,20}$ including only chordoma patients. The median dose was 68.4-77.4 RBE, most commonly given in 1.8-2 RBE fractions. LC rates were 36\%-98\% (1 year), 18\%-90\% (2 years), $84 \%-97 \%$ (3 years), $62 \%-85 \%$ (5 years), and $49 \%-$ $58 \%$ (10 years). The OS rates were 93\%-96\% (2 years), $87 \%-92 \%$ (3 years), $78 \%-87 \%$ (5 years), and $53 \%-63 \%$ (10 years). PFS was only reported by Chen et al., ${ }^{13}$ who reported $90 \%$ at 5 years and $80 \%$ at 10 years. DFS was $68 \%-77 \%$ at 3 years and $61 \%-72 \%$ at 5 years. Metastases occurred in $12 \%-14 \%$ (3 years), $20 \%-27 \%$ (5 years), and $8 \%-24 \%$ (LFU). Acute toxicities occurred in $84 \%-100 \%$ (2\%-16\% suffered toxicities $\geq$ CTC grade 3$)$, and late tox- icities occurred in 10\%-100\% (0\%-28\% suffered toxicities $\geq$ CTC grade 3). Chen et al..$^{13}$ and Kabolizadeh et al. ${ }^{19}$ described nonsurgical patients. Both cohorts were from the MGH and used a median dose of 77.4 RBE delivered in 1.8-2 RBE fractions. At 3 years, the OS was $89 \%-92 \%$ and DSS was $96 \%-97 \%$. Metastasis was seen in 12\%-14\% and the OS at 5 years was $78 \%-82 \%$.

\section{CIRT Series}

Fourteen studies described CIRT. ${ }^{21,23-32,34-36}$ Studies were small (7-219 patients), with only 2 studies from the National Institute of Radiological Sciences in Chiba, Japan, including more than 100 patients. ${ }^{23,27}$ The median age was 54-67 years, patients were 48\%-80\% male, and the median follow-up was 18-80 months. Except in the study by Matsumoto et al., ${ }^{47}$ in all treated patients $(94 \%-100 \%)$ chordoma was the most common disease, with 12 studies including only chordoma patients. ${ }^{21,23-27,29,30,32,34-36}$ The median dose was 64-86 RBE given in 2.2-4.6 RBE fractions. LC rates were 90\%-94\% (1 year), $76 \%-85 \%$ (2 years), $53 \%-95 \%$ ( 3 years), and $53 \%-100 \%$ (5 years). The OS rates were $97 \%-100 \%$ (2 years), $59 \%-86 \%$ (3 years), and $52 \%-86 \%$ (5 years). The PFS rates were $90 \%-95 \%(1$ year), $70 \%-80 \%$ ( 2 years), $48 \%-76 \%$ (3 years), and $48 \%-$ $94 \%$ (10 years). The DFS was $50 \%-53 \%$ at 5 years, and Imai et al. ${ }^{27}$ reported a 10 -year DFS of $31.3 \%$. Metastases occurred in $7 \%-9 \%$ (1 year), 32\%-52\% (5 years), and $2 \%-39 \%$ (LFU). Acute toxicities occurred in 26\%-48\% (0\%-18\% suffered CTC grade $\geq 3$ events), and late toxicities occurred in 59\%-89\% (0\%-21\% suffered CTC grade $\geq 3$ events). Eight studies examined strictly nonsurgical patients, ${ }^{23,26-30,32,35}$ and in 6 of these studies patients were 


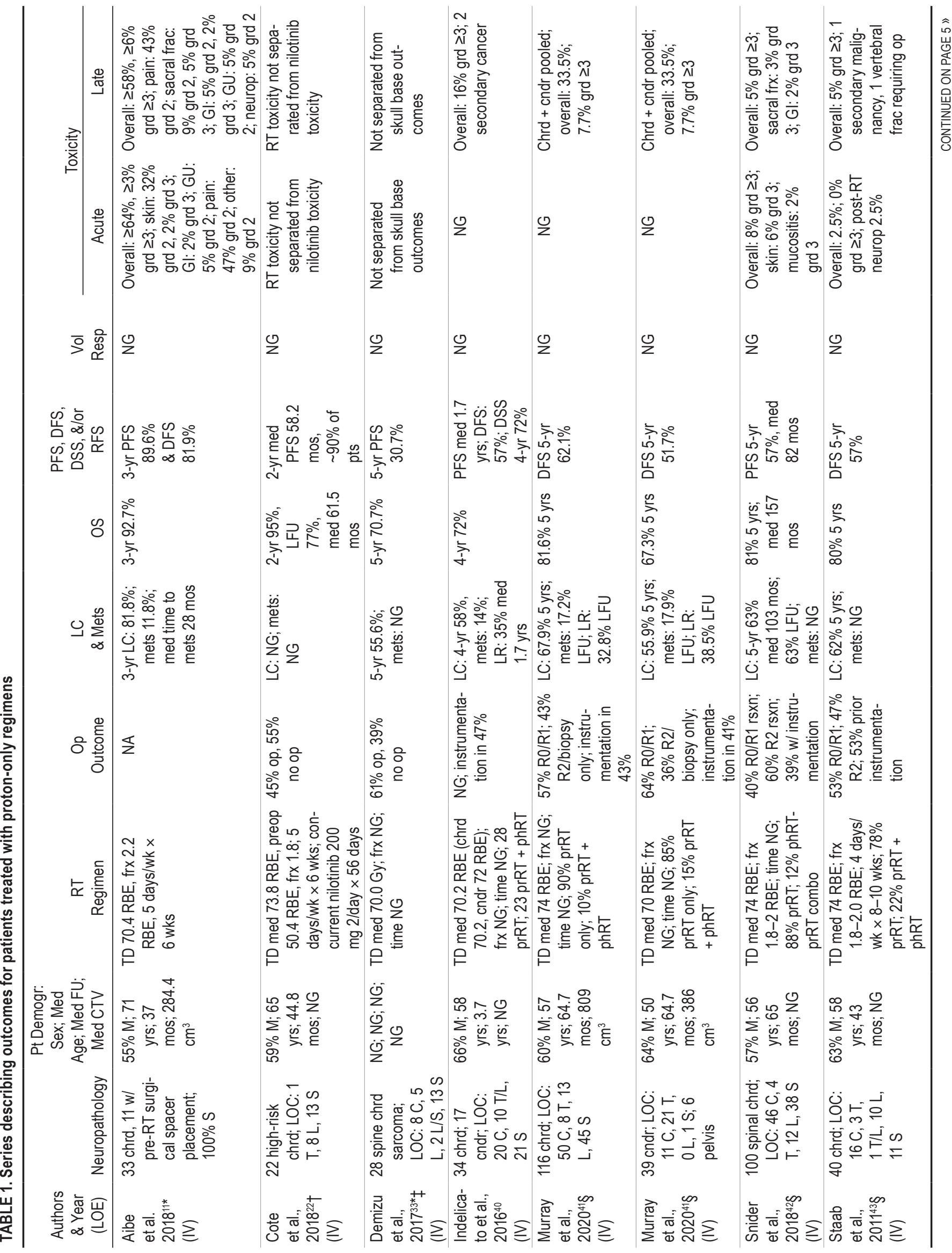




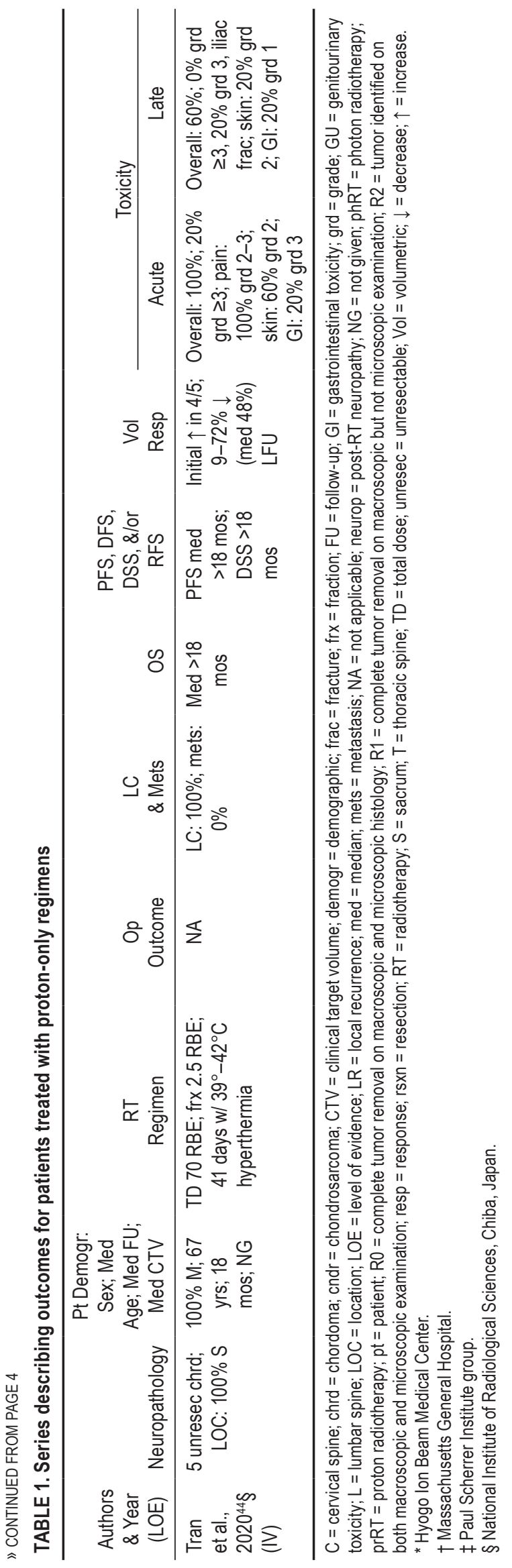

treated at the National Institute of Radiological Sciences, ${ }^{23,26-29,32}$ so much of the data from these sources were from a similar and likely overlapping set of nonsurgical patients treated at the National Institute of Radiological Sciences. Five-year outcomes were LC $72 \%-100 \%$, OS $52 \%-86 \%$, and DFS 50\%-53\%. Metastasis occurred in $14 \%-36 \%$ by the LFU. ${ }^{23,26-30,32,35}$

\section{Other Series}

In the 3 remaining series, 2 reported pooled outcomes for patients treated with proton therapy and CIRT, ${ }^{37,39}$ and 1 series described patients treated with helium, neon, or combination helium/neon-based therapies. ${ }^{38}$ All 3 series studied sacral chordoma; the median patient age was 6172 years, and $48 \%-86 \%$ of patients were male. The median dose was 70.4-79.2 RBE given in fractions of 2-4.4 RBE over a period of 4 weeks to 61 days. Only 5-year OS (85\%-100\%) and rates of metastases at the LFU (14\%$29 \%$ ) were reported by more than 1 study. ${ }^{38,39}$ Both studies from the Hyogo Ion Beam Medical Center employed a surgical spacer implanted posterior to the rectum to reduce dosing to the anus and rectum, whereas the study of Schoenthaler et al..$^{38}$ treated a mixture of surgical and nonsurgical patients. Only Tsugawa et al..$^{39}$ reported outcomes for nonsurgical patients.

\section{Discussion}

Recent evidence has begun to suggest that adjuvant radiation ${ }^{9}$ may be the most significant predictor of LC in primary sarcomas of the spine and sacrum. The reasons for this are at present unknown; however, preliminary histology-based studies suggest it may be due to micrometastatic disease outside of the tumor capsule. ${ }^{48}$ Additionally, some lesions are not amenable to surgery, meaning that definitive radiotherapy may be the only option. Given the radioresistance of primary bone sarcomas to conventional radiation, there is great interest in the utility of charged-particle therapies for the definitive or adjuvant management of these lesions. Previous reviews focusing on chordoma and chondrosarcoma of the skull base have found these modalities to produce high rates of OS and LC of up to 5 years. Here, we provide a similar analysis of the efficacy of these treatment methods in the mobile spine and sacrum. Relative to the reported rates for skull base lesions, we find that OS and LC rates are lower. However, given the findings from population-level studies, ${ }^{49}$ it is possible that these differences may be due to the overall better prognosis of skull base lesions relative to those of the spine/sacrum.

Of particular interest are the reported efficacies of the various radiation modalities as definitive therapies. The available data are severely limited. Of those studies including only nonsurgical patients, $\mathrm{LC}$ is similar for patients treated with protons $(82 \% \text { at } 3 \text { years) })^{11}$ and CIRT $(72 \%-$ $100 \%$ at 5 years). OS is likewise similar for combined photon/proton radiotherapy and CIRT at 5 years $(78 \%-82 \%$ vs $52 \%-86 \%$ ). Of note, statistical comparisons were not possible given the significant overlap in the reported study populations. Despite this, Mima et al. ${ }^{37}$ directly compared outcomes between proton and hadron therapy for sacral chordoma and saw no significant differences in LC, PFS, 


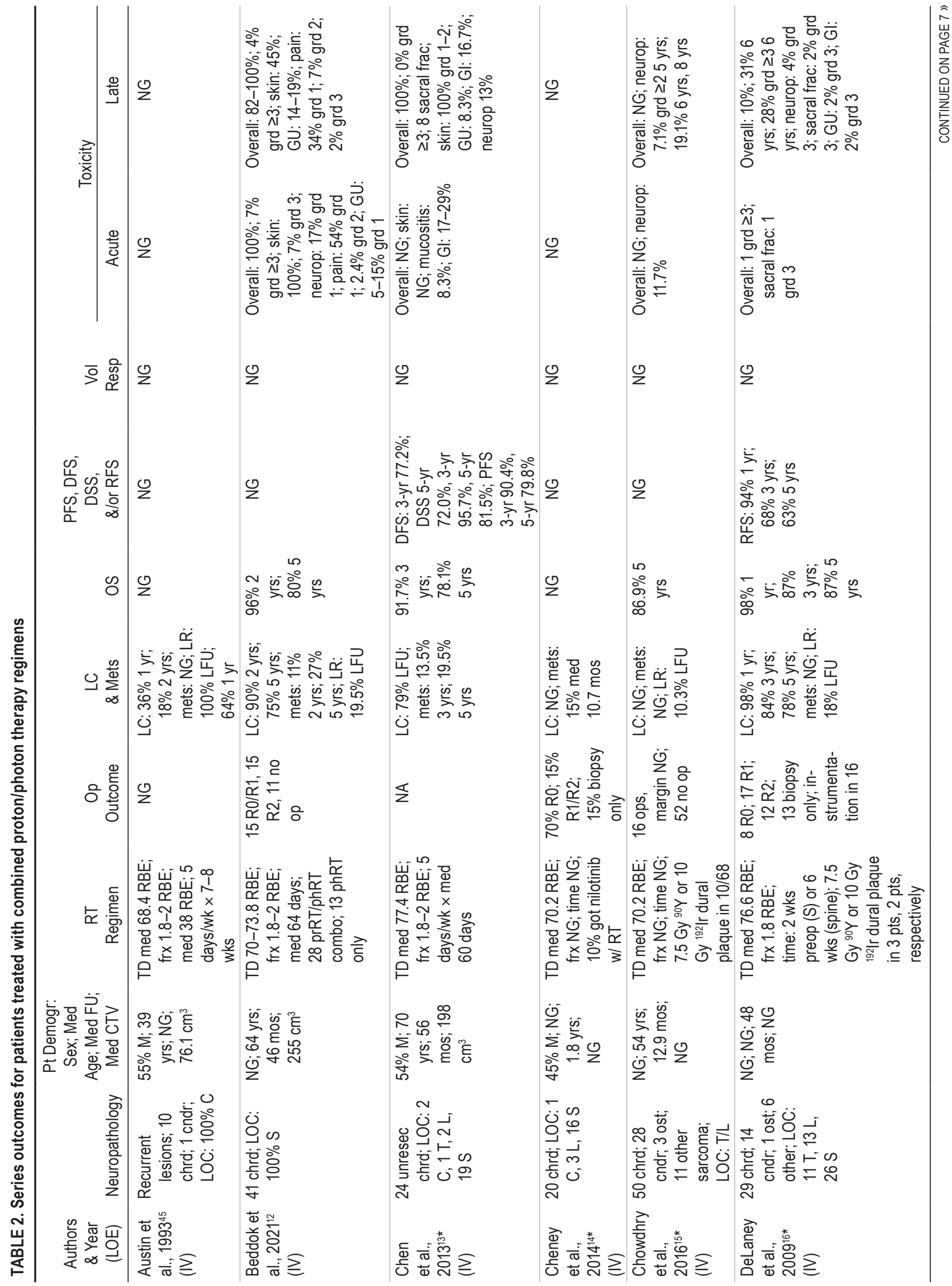



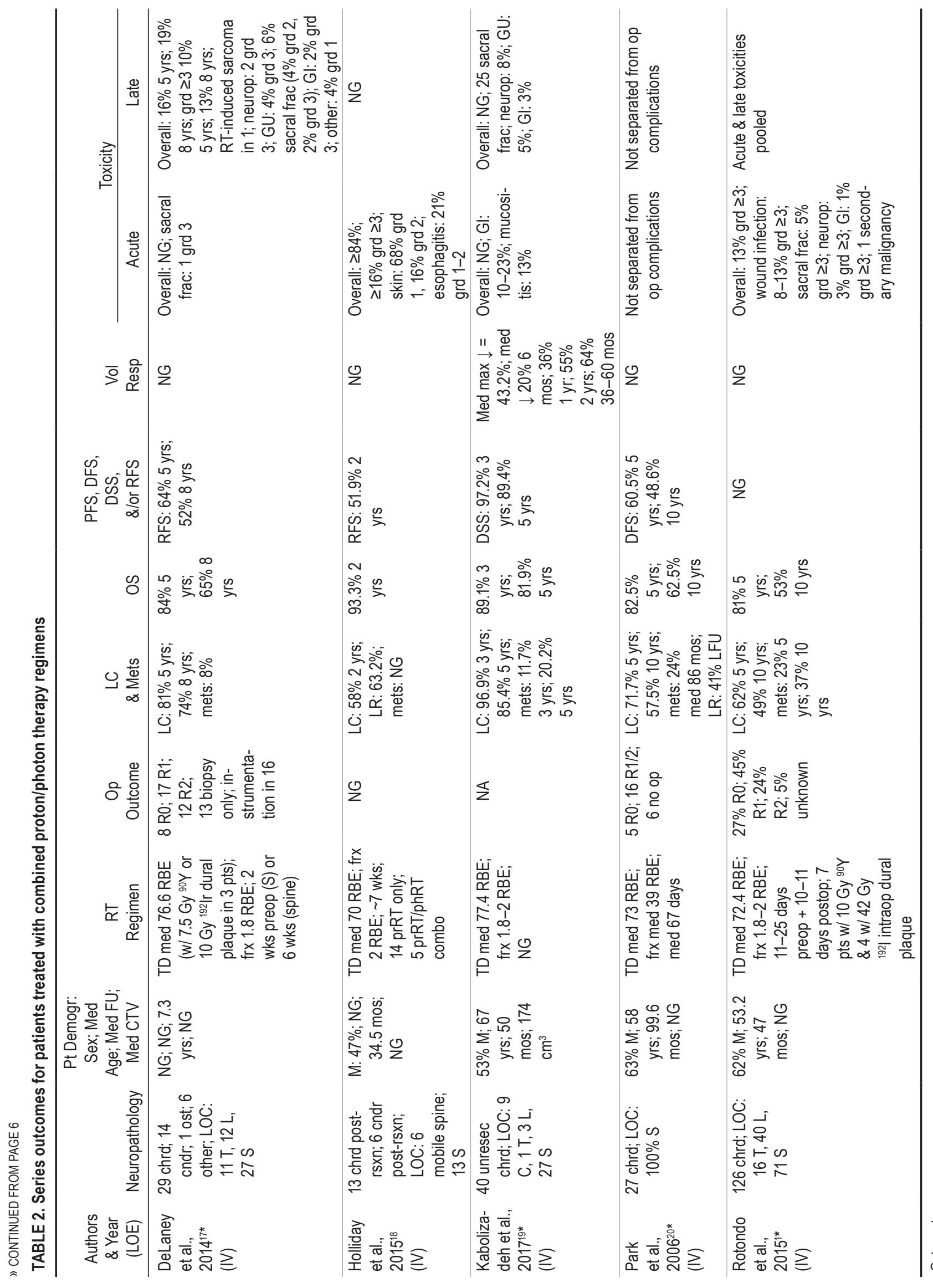

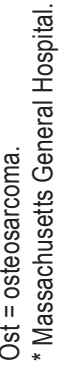




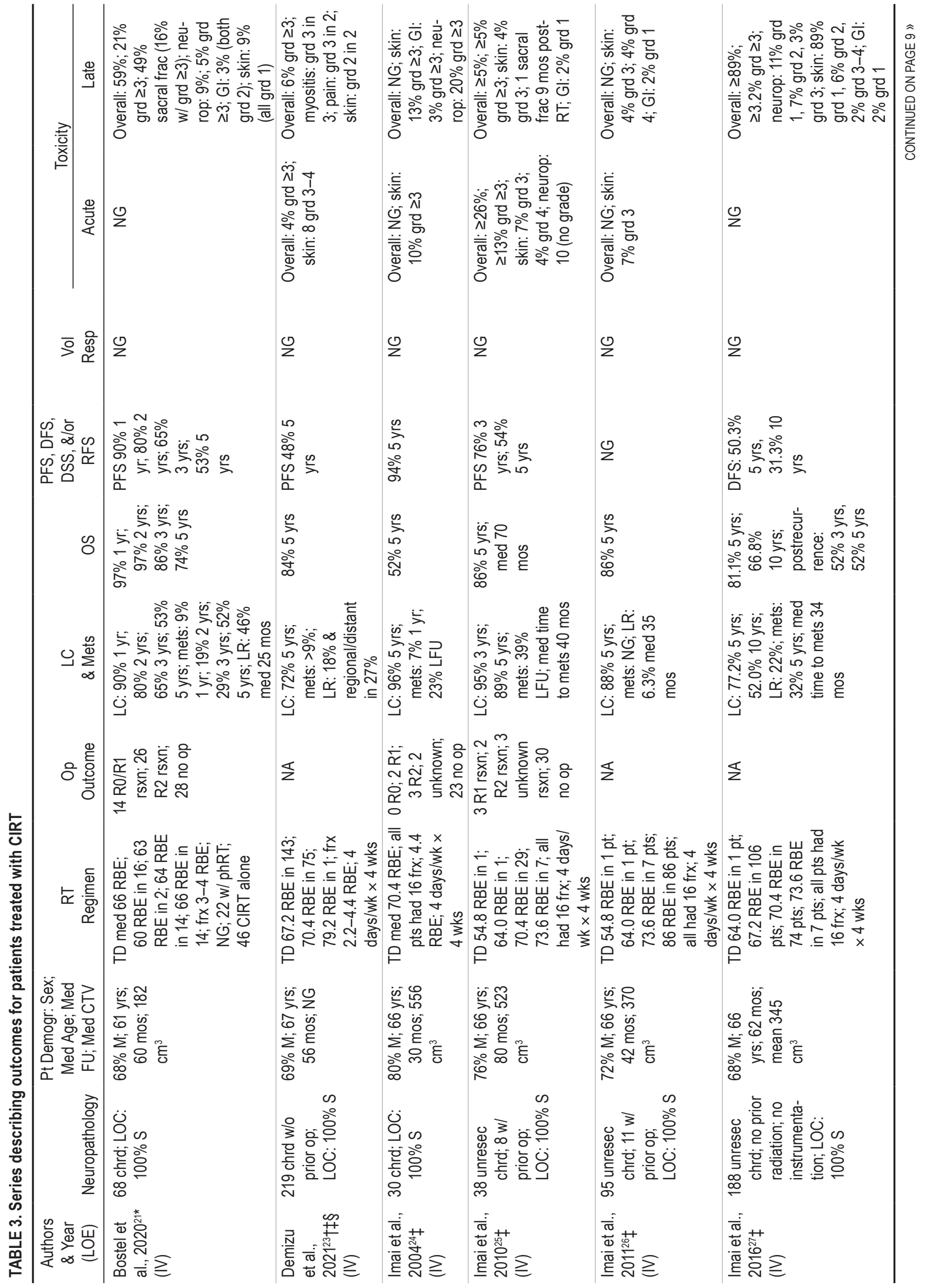




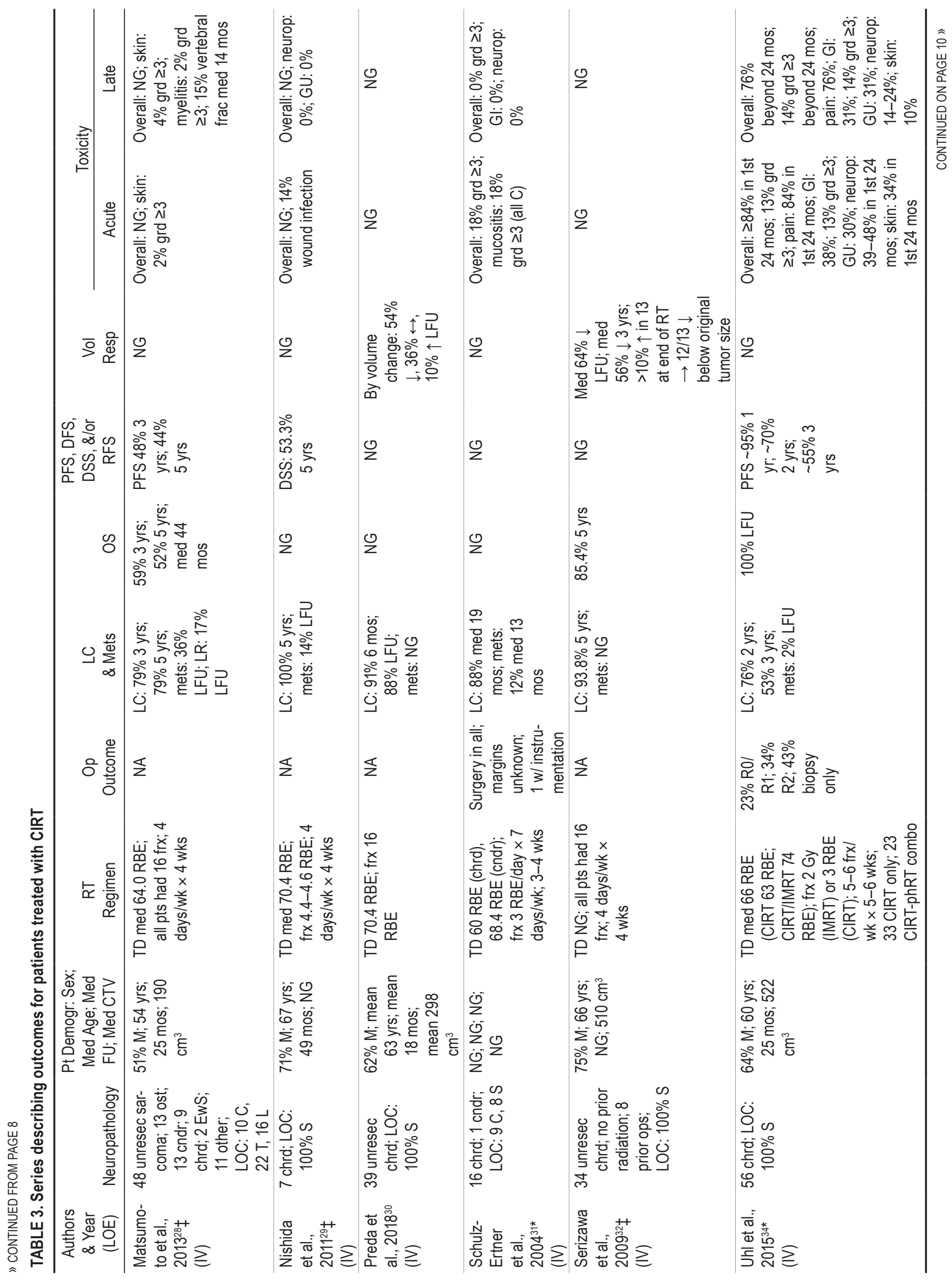




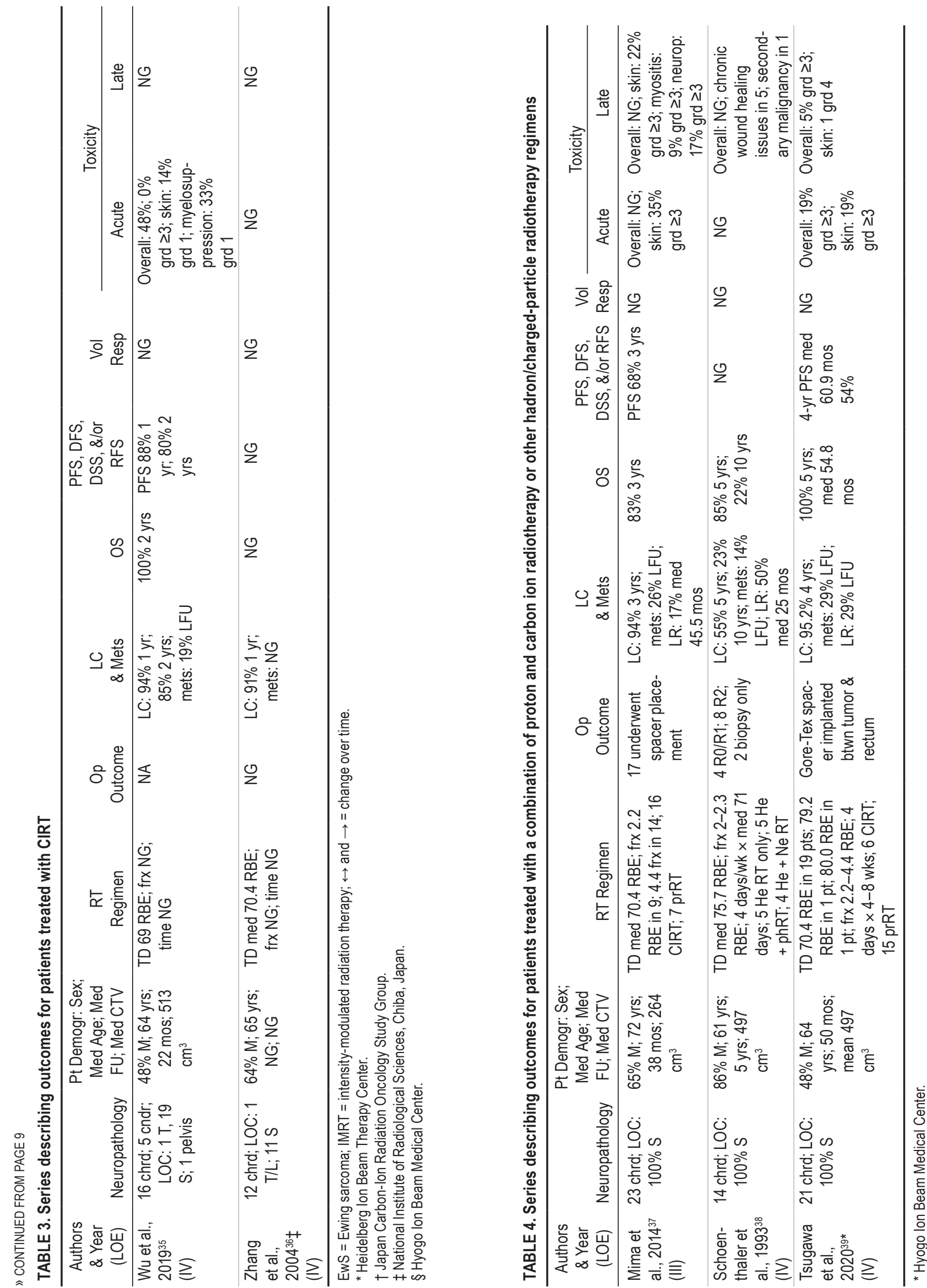


TABLE 5. Dose safety limits for radiation planning in proton and charged-particle therapy

\begin{tabular}{|c|c|c|c|c|c|c|}
\hline \multirow[b]{2}{*}{ Location } & \multicolumn{6}{|c|}{ Institution } \\
\hline & HbIBTC & HylBMC & Institut Curie & MGH & PSI & Shanghai Group \\
\hline \multicolumn{7}{|l|}{ Nervous system } \\
\hline Optic nerves & $\leq 54 \mathrm{~Gy}$ & - & - & - & $\leq 60 \mathrm{~Gy}$ & - \\
\hline Brainstem & $\begin{array}{l}\leq 60 \text { Gy (surface); } \\
\quad \leq 50 \text { Gy (center) }\end{array}$ & - & - & - & $<63$ Gy & - \\
\hline Spinal cord & $\leq 45 \mathrm{~Gy}$ & $\leq 48.0 \mathrm{~Gy}$ & - & - & - & $\leq 40 \mathrm{~Gy}$ \\
\hline Surface & - & - & $\leq 54$ Gy; $\leq 45$ Gy (posterior edge) & $\leq 63 \mathrm{~Gy}$ & $\leq 63 \mathrm{~Gy}$ & - \\
\hline Core & - & - & $\leq 48 \mathrm{~Gy}$ & $\leq 54 \mathrm{~Gy}$ & $\leq 54 \mathrm{~Gy}$ & - \\
\hline Cauda equina & - & - & $\leq 57$ Gy (male); $\leq 67$ Gy (female) & - & $\leq 64 \mathrm{~Gy}$ & - \\
\hline Skin & - & $\begin{array}{l}\leq 90 \% \text { receiving } \\
\geq 63.0 \text { Gy }\end{array}$ & $\leq 64$ Gy; $\leq 60$ Gy (gluteal fold) & $\leq 66 \mathrm{~Gy}$ & - & - \\
\hline \multicolumn{7}{|l|}{ Thoracic viscera } \\
\hline Heart & - & - & - & - & $\begin{array}{l}\leq 2 / 3 \text { of organ }(\leq 30 \mathrm{~Gy}) ; \\
\leq 1 / 3 \text { of } \operatorname{organ}(\leq 40 \mathrm{~Gy})\end{array}$ & $\leq 40 \mathrm{~Gy}$ \\
\hline Lung & - & - & - & - & $\begin{array}{l}\leq 2 / 3 \text { of organ }(\leq 30 \mathrm{~Gy}) ; \\
\leq 1 / 3 \text { of organ }(\leq 40 \mathrm{~Gy})\end{array}$ & $\leq 40 \mathrm{~Gy}$ \\
\hline \multicolumn{7}{|l|}{ Abdominal viscera } \\
\hline Kidney & - & - & - & - & $\leq 20 \mathrm{~Gy}$ & - \\
\hline Small intestine & $\leq 50 \mathrm{~Gy}$ & $\leq 52.0 \mathrm{~Gy}$ & - & - & - & - \\
\hline Large intestine & - & $\leq 57.0 \mathrm{~Gy}$ & $\leq 60 \mathrm{~Gy}$ & - & - & - \\
\hline Rectum & $\begin{array}{r}\leq 70 \text { Gy; } \leq 30 \text { Gy } \\
\text { (anterior third) }\end{array}$ & $\begin{array}{c}\leq 17.0 \% \text { pts wl } \\
\geq 65.0 \mathrm{~Gy}\end{array}$ & $\leq 60 \mathrm{~Gy}$ & - & - & $\leq 66 \mathrm{~Gy}$ \\
\hline \multicolumn{7}{|l|}{ Genitalia } \\
\hline Penis & - & - & $\leq 58 \mathrm{~Gy}$ & - & - & - \\
\hline Testis & - & - & $\leq 2 \mathrm{~Gy}$ & - & - & - \\
\hline Ovaries & - & - & $\leq 5 \mathrm{~Gy}$ & - & - & - \\
\hline
\end{tabular}

HbIBTC = Heidelberg Ion Beam Therapy Center; HyIBMC = Hyogo Ion Beam Medical Center; PSI = Paul Scherrer Institute.

or OS. Investigations comparing combined proton/photon therapy to photon-only therapy have suggested that the addition of charged particles may improve LC. ${ }^{17}$ Importantly, LC and OS appear similar between patients treated with definitive charged-particle (proton or hadron-based) radiotherapy and historical multicenter surgical cohorts of chordoma (LC approximately $75 \%-80 \%$ and OS approximately $70 \%-85 \%$ at 5 years following R0 resection) ${ }^{5}$ and other sarcomas. ${ }^{4,50}$

\section{Timing of Radiation}

Technical concerns related to the use of adjuvant radiotherapy include radiation planning and monitoring of tumor response. The first consideration is whether radiation should be given presurgery, postsurgery, or both. Based on the MGH experience, ${ }^{1}$ LC appears superior in surgically treated patients who receive pre- and postoperative radiation versus postoperative radiation alone. Notably, this finding was independent of the total radiation dose administered. However, many investigators express concerns about the negative impact of neoadjuvant radiotherapy on surgical wound healing. To this end, a recent survey of spine oncology specialists found that the minority routinely recommend preoperative radiotherapy for either primary or recurrent chordoma. ${ }^{51}$
In light of the above findings regarding the more common preference for postoperative treatment, the prevailing inquiry remains in whether the timing of postoperative radiotherapy matters. In their series from MD Anderson, Holliday et al..$^{18}$ found that better LC was achieved with early versus late adjuvant proton radiotherapy (relapsefree survival [RFS] $75 \%$ vs $45 \%$ at 2 years). In line with these results, the above survey found that most experts recommend radiotherapy within 8 weeks of surgery. ${ }^{51}$

\section{Radiation Planning}

Dosing schemas for adjuvant charged-particle therapy have been described by several centers, including the Paul Scherrer Institute, ${ }^{42,43}$ the Hyogo Ion Beam Medical Center in Japan,,${ }^{11,39}$ and the MGH. ${ }^{19}$ These schemas are based on the desire to minimize irradiation of the spinal cord, nerve roots, and adjacent healthy tissues (Table 5). In general, most groups recommend the following limits: brainstem ( $\leq 63$ Gy) and spinal cord (surface $\leq 63$ and core $\leq 48-54$ $\mathrm{Gy}^{11,12,15-17,21,31,37}$. Some groups ${ }^{35}$ are even more conservative and recommend that doses to healthy tissues not exceed $40 \mathrm{~Gy}$. These recommendations are consistent with the evidence as recently reviewed by Kirkpatrick et al..$^{52}$ The authors found a dose-response relationship between spinal cord irradiation and injury, with doses of 54 Gy cor- 
responding to a $<1 \%$ risk of injury compared with a nearly $10 \%$ risk for doses of $61 \mathrm{~Gy}$.

Skin doses are also minimized to reduce the rates of radiation-induced dermatitis, though this complication occurs in up to $100 \%$ of patients in the acute setting. ${ }^{12}$ These skin toxicities are likely of increased importance in postoperative patients, as irradiation is a known risk factor for wound infection in spine oncology patients. ${ }^{53}$ However, given that some recent evidence suggests that radiation may more strongly dictate LC than surgical margins, ${ }^{9}$ many spinal oncologists recommend its use..$^{51}$ As alluded to by the aforementioned survey, there is no high-quality evidence on what radiotherapy time frame provides the best LC while minimizing results. Yet, based on a recent review of level III/IV data in the spinal metastasis population, 2 weeks postoperatively may be an ideal time point, ${ }^{53}$ in line with the results of the Dea et al. survey. ${ }^{51}$ Additionally, using smaller fractions may help reduce skin toxicities, a finding that the Hyogo group ${ }^{39}$ cited as a primary reason for shifting from fractions of 4.4 to $2.2 \mathrm{RBE}$. Nevertheless, some groups continue with 4.4-4.6 RBE fractions because they feel it may offer superior LC. ${ }^{26}$

The guidelines for isodose line contouring are beyond the scope of this study, as is the best timing of radiographic follow-up. However, the included studies suggested leaving a minimum margin around the spinal cord of 3-5 $\mathrm{mm}^{21}$ or, in most cases, 5-10 mm. ${ }^{11,35,39,42,43}$ Experts at the MGH have recommended even larger margins, $1-1.5 \mathrm{~cm}$ around the spinal cord and $3.5 \mathrm{~cm}$ along neurovascular bundles. ${ }^{19}$ These large margins may negatively impact the ability to treat the entire tumor penumbra, however, and so the surgeon should discuss with the patient the relative benefits of greater tumor bed coverage relative to the increased risk of postradiation neuropathy. The group from the Chiba Heavy Ion Medical Accelerator Center report that the risk of neural injury may only be significant for high doses, as evidenced by their finding that postradiation sciatic nerve injury was only a significant risk for those receiving $>70$ Gy to $>10 \mathrm{~cm}$ of the sciatic nerve.

\section{Theoretical Advantages of Charged-Particle Therapy Over Photon Radiotherapy}

The primary theoretical advantage of using charged particles is the ability to deliver high-dose radiation to target tissues while minimizing doses to normal tissues. This property derives from the higher mass of the charged particles and is described by the Bragg peak displayed by charged-particle therapies (Fig. 2) ${ }^{54}$ Some clinical studies have supported the superiority of charged-particle therapies. In a series of 41 patients with sacral chordoma undergoing definitive or adjuvant radiotherapy, Beddok et al. ${ }^{12}$ showed that those treated with combined proton/photon tomotherapy had $75 \%$ lower doses to the bladder than those treated with photons alone.

Other evidence, however, has failed to demonstrate the superiority of charged particles. In a multicenter study by the Sacral Tumor Society working group, ${ }^{55}$ the use of proton versus photon radiotherapy was not associated with differences in OS, DFS, DSS, or rates of metastasis. Yet patients treated with protons versus photons suffered higher rates of wound complication and stress fracture, in-

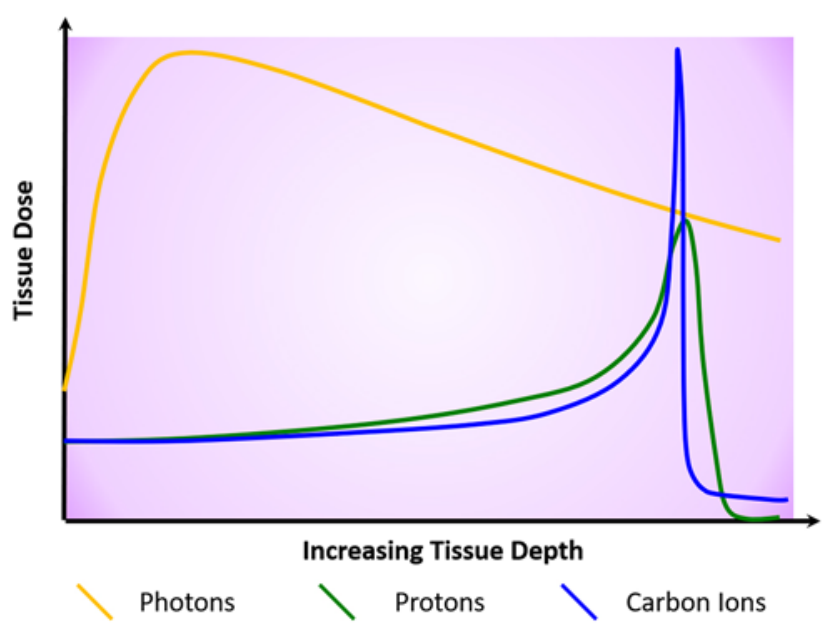

FIG. 2. Plot illustrating dose delivery as a function of depth of tissue penetrated for three radiation modalities. Charged-particle therapies, such as protons and carbon ions, demonstrate a Bragg peak, where there is a steep rise in the delivered dose at the beam's focus point and a commensurately steep decline in dose delivered to those tissues past the tissue target. This behavior theoretically enables charged particles to deliver high doses to target tissue with relatively little radiation delivered to the surrounding healthy tissues. The overall tissue penetration of charged particles is determined by their initial energy (in megaelectron volts, MeV), the material/tissue through which they are passing, and the type of particles used (e.g., carbon ions).

dependent of the radiotherapy dose, tumor size, and use of high versus low sacral resection. Snider et al. ${ }^{42}$ similarly found no difference in LC, PFS, or OS between patients treated with proton radiotherapy and those treated with combined proton/focused photon radiotherapy. Only $12 \%$ of the patients received combination therapy, however. Beddok et al. ${ }^{12}$ likewise saw no difference in OS between tomotherapy-treated and combined proton/tomotherapytreated patients. Locoregional recurrence appeared to be lower in the combined proton/photon treatment group, but the difference was not significant. Indelicato et al. ${ }^{40}$ similarly found a nonsignificant trend toward better LC in the proton/photon group. Beddok et al. ${ }^{12}$ found that proctitis and acute cauda equina syndrome were more common in the tomotherapy group, but dermatitis was more common in the proton/tomotherapy combination group, as was posttreatment pain (acute phase).

In light of these findings, it is unclear that charged particles are superior to modern photon-based modalities. To this end, Jin et al..$^{56}$ recently reported LC rates of $96.3 \%$ and $89.9 \%$ at 3 and 5 years, respectively, in patients with chordoma of the mobile spine or sacrum treated with 24Gy single-fraction radiosurgery delivered as either adjuvant or definitive treatment. These rates are comparable or superior to the rates reported here and in historical cohorts, suggesting that charged particles may not be superior to modern focused radiation modalities. Additional studies directly comparing the outcomes of these two modalities are necessary.

\section{Instrumentation and Radiotherapy}

Many patients treated for primary spinal or sacral sar- 
comas require instrumentation to correct iatrogenic instability, which is most common in patients treated with high sacrectomy or en bloc spondylectomy. However, in reviewing the series here, we note that postradiation sacral and vertebral fracture occur in up to $49 \%$ of patients, with as many as $16 \%$ having fractures severe enough to require intervention. . $^{11,13,19,21,28,42}$ This may be due to the trabecular bone loss that is linked to the use of high-dose radiation. ${ }^{57}$ Consequently, many additional patients may require instrumentation.

Postoperative radiotherapy and follow-up are complicated by placement of metallic instrumentation, which may generate image artifacts that decrease the accuracy with which isodose lines can be drawn and cause local radiation scattering that often forces radiation oncologists to use lower prescribed doses.$^{40}$ Consistent with these findings, data from the Paul Scherrer Institute ${ }^{41-43}$ indicate that LC, PFS, and OS were all much poorer for patients receiving instrumentation. According to an analysis by Snider et al., ${ }^{42}$ the influence of instrumentation remained significant for LC and PFS on multivariable analysis, and in a study by Murray et al..$^{41}$ the influence of instrumentation remained significant for OS and LC; it was of borderline significance for PFS. At the MGH, DeLaney et al. ${ }^{16}$ similarly found a more than 2.5-fold higher rate of local recurrence in spine sarcoma patients with instrumentation. However, the difference did not meet the threshold for significance, perhaps due to the relatively small sample size. Of note, Staab et al. ${ }^{43}$ found that the negative effects of instrumentation on radiotherapy were greatest for those in most need of adjuvant radiotherapy; the decrements in LC were greatest for patients with gross residual disease at the time of radiotherapy.

These difficulties with radiotherapy planning along with the difficulty that instrumentation creates in executing effective radiographic follow-up have led some centers to consider the use of carbon fiber-reinforced polyetheretherketone (CFR-PEEK) instrumentation. ${ }^{58,59}$ CFR-PEEK rods, unlike titanium rods, are radiolucent and so create minimal artifact on the $\mathrm{CT}$ scans employed for radiation planning. ${ }^{60,61}$ Additionally, CFR-PEEK rods better approximate the elastic modulus of cortical bone. ${ }^{62}$ However, they have been found in cadaveric models to produce less-rigid constructs. ${ }^{63}$ No differences in hardware complications have been noted in early clinical series; ${ }^{59}$ however, followup is extremely limited at present and further investigation is merited.

\section{Study Limitations}

The present study has inherent limitations, several of which have potential implications for its interpretation. The most notable limitation is that all included studies are level III or IV data. No studies directly compared followup between photon- and charged-particle-treated patients. Additionally, the majority of reports are based on limited experiences reported by a half dozen centers, so it is unclear whether the present results are generalizable to all patients with chordomas and sarcomas of the spine and sacrum. To this end, a quantitative meta-analysis could not be performed because of the significant overlap in study samples and the limited study quality. A further limita- tion was the overlap in the discussed pathologies, as the outcomes for chordomas, chondrosarcomas, and other sarcomas were grouped in most series, whereas previous work has indicated that prognoses differ for these pathologies. ${ }^{4,5,50,64,65}$ Similarly, for chondrosarcomas ${ }^{66}$ and osteosarcomas, ${ }^{67}$ tumor grade and subtype have a significant impact on both OS and DSS and the radiation responsiveness of the lesion. Additionally, research published in the past year has suggested that tumor grade has a significant impact on radiation responsiveness in chondrosarcoma. ${ }^{68}$ To better appreciate the relative influence of charged-particle therapies on LC and OS, it will be necessary to incorporate these factors. Last, from a practical standpoint, proton treatment is offered in relatively few centers in the US, which may limit the feasibility of this treatment for patients from some geographic regions. Carbon ion treatment is even rarer, and no centers in the US currently offer this treatment. Consequently, it is unclear as to whether the present results can be incorporated into treatment protocols for all centers.

\section{Conclusions}

This review of the literature demonstrated that the current data evidence for proton and carbon ion radiotherapy for chordoma and sarcomas of the mobile spine and sacrum is quite limited and derived from a small number of centers. Given the quality of data, this systematic review does not demonstrate the superiority of one specific chargedparticle approach in the definitive or adjuvant setting. In the adjuvant setting, charged-particle therapy likely offers similar LC to modern photon therapy regimens. Likewise, comparing reports of definitive charged-particle therapy for nonsurgical patients to historical surgical cohorts suggests that PFS may be similar for those treated with proton and carbon ion therapy. Additional investigations based on experiences from a larger number of centers are required.

\section{Appendix}

\section{Search Queries for the PubMed, Embase, OVID Medline, and Web of Science Databases}

PubMed Query: ("chordoma" OR "chondrosarcoma” OR "osteosarcoma" OR "osteogenic sarcoma" OR "Ewing sarcoma" OR "Ewing's sarcoma” OR "Ewings sarcoma" OR "primary bone tumor" OR "primary vertebral tumor" OR "primary spine tumor") AND (spine OR spinal OR vertebral OR vertebra OR vertebrae OR sacrum OR sacral) AND ("carbon ion" OR "hadron" OR "proton" OR "proton therapy" OR "carbon ion therapy" OR "hadron therapy" OR "charged ion" OR "charged ion therapy")

Embase Query: ('chordoma' OR ‘chondrosarcoma' OR ‘osteosarcoma' OR ‘osteogenic sarcoma' OR 'ewing sarcoma' OR 'ewings sarcoma' OR 'primary bone tumor' OR 'primary vertebral tumor' OR 'primary spine tumor') AND (spine OR spinal OR vertebral OR vertebra OR vertebrae OR sacrum OR sacral) AND ('carbon ion' OR 'hadron' OR ‘proton' OR 'proton therapy' OR 'carbon ion therapy' OR 'hadron therapy' OR 'charged ion' OR 'charged ion therapy')

OVID Medline Query: (("chordoma" or "chondrosarcoma" or "osteosarcoma" or "osteogenic sarcoma" or "Ewing sarcoma" or "Ewing's sarcoma" or "Ewings sarcoma" or "primary bone tumor" or "primary vertebral tumor" or "primary spine tumor") and (spine or spinal or vertebral or vertebra or vertebrae or 
sacrum or sacral) and ("carbon ion" or "hadron" or "proton" or "proton therapy" or "carbon ion therapy" or "hadron therapy" or "charged ion" or "charged ion therapy")).mp. [mp=title, abstract, original title, name of substance word, subject heading word, floating sub-heading word, keyword heading word, organism supplementary concept word, protocol supplementary concept word, rare disease supplementary concept word, unique identifier, synonyms]

Web of Science Query: ALL=("chordoma" OR "chondrosarcoma" OR "osteosarcoma" OR "osteogenic sarcoma" OR "Ewing sarcoma" OR "Ewing's sarcoma" OR "Ewings sarcoma" OR "primary bone tumor" OR "primary vertebral tumor" OR "primary spine tumor") AND ALL=(spine OR spinal OR vertebral OR vertebra OR vertebrae OR sacrum OR sacral) AND ALL=("carbon ion" OR "hadron" OR "proton" OR "proton therapy" OR "carbon ion therapy" OR "hadron therapy" OR "charged ion" OR "charged ion therapy")

\section{References}

1. Rotondo RL, Folkert W, Liebsch NJ, et al. High-dose protonbased radiation therapy in the management of spine chordomas: outcomes and clinicopathological prognostic factors. $J$ Neurosurg Spine. 2015;23(6):788-797.

2. Yamazaki T, McLoughlin GS, Patel S, et al. Feasibility and safety of en bloc resection for primary spine tumors: a systematic review by the Spine Oncology Study Group. Spine (Phila Pa 1976). 2009;34(22)(suppl):S31-S38.

3. Amendola L, Cappuccio M, De Iure F, et al. En bloc resections for primary spinal tumors in 20 years of experience: effectiveness and safety. Spine J. 2014;14(11):2608-2617.

4. Dekutoski MB, Clarke MJ, Rose P, et al. Osteosarcoma of the spine: prognostic variables for local recurrence and overall survival, a multicenter ambispective study. J Neurosurg Spine. 2016;25(1):59-68.

5. Gokaslan ZL, Zadnik PL, Sciubba DM, et al. Mobile spine chordoma: results of 166 patients from the AOSpine Knowledge Forum Tumor database. J Neurosurg Spine. 2016;24(4): 644-651.

6. Kerr DL, Dial BL, Lazarides AL, et al. Epidemiologic and survival trends in adult primary bone tumors of the spine. Spine J. 2019;19(12):1941-1949.

7. Rich TA, Schiller A, Suit HD, Mankin HJ. Clinical and pathologic review of 48 cases of chordoma. Cancer. 1985; 56(1):182-187.

8. Suit H, Goitein M, Munzenrider J, et al. Evaluation of the clinical applicability of proton beams in definitive fractionated radiation therapy. Int J Radiat Oncol Biol Phys. 1982; 8(12):2199-2205.

9. van Wulfften Palthe ODR, Tromp I, Ferreira A, et al. Sacral chordoma: a clinical review of 101 cases with 30-year experience in a single institution. Spine J. 2019;19(5):869-879.

10. Liberati A, Altman DG, Tetzlaff J, et al. The PRISMA statement for reporting systematic reviews and meta-analyses of studies that evaluate healthcare interventions: explanation and elaboration. BMJ. 2009;339:b2700.

11. Aibe N, Demizu Y, Sulaiman NS, et al. Outcomes of patients with primary sacral chordoma treated with definitive proton beam therapy. Int J Radiat Oncol Biol Phys. 2018;100(4): 972-979.

12. Beddok A, Saint-Martin C, Mammar H, et al. High-dose proton therapy and tomotherapy for the treatment of sacral chordoma: a retrospective monocentric study. Acta Oncol. 2021;60(2):245-251.

13. Chen YL, Liebsch N, Kobayashi W, et al. Definitive highdose photon/proton radiotherapy for unresected mobile spine and sacral chordomas. Spine (Phila Pa 1976). 2013;38(15): E930-E936.

14. Cheney MD, Chen YL, Lim R, et al. [18F]-Fluoromisonida- zole positron emission tomography/computed tomography visualization of tumor hypoxia in patients with chordoma of the mobile and sacrococcygeal spine. Int J Radiat Oncol Biol Phys. 2014;90(5):1030-1036.

15. Chowdhry VK, Liu L, Goldberg S, et al. Thoracolumbar spinal cord tolerance to high dose conformal proton-photon radiation therapy. Radiother Oncol. 2016;119(1):35-39.

16. DeLaney TF, Liebsch NJ, Pedlow FX, et al. Phase II study of high-dose photon/proton radiotherapy in the management of spine sarcomas. Int J Radiat Oncol Biol Phys. 2009;74(3): 732-739.

17. DeLaney TF, Liebsch NJ, Pedlow FX, et al. Long-term results of Phase II study of high dose photon/proton radiotherapy in the management of spine chordomas, chondrosarcomas, and other sarcomas. J Surg Oncol. 2014;110(2):115-122.

18. Holliday EB, Mitra HS, Somerson JS, et al. Postoperative proton therapy for chordomas and chondrosarcomas of the spine: adjuvant versus salvage radiation therapy. Spine (Phila Pa 1976). 2015;40(8):544-549.

19. Kabolizadeh P, Chen YL, Liebsch N, et al. Updated outcome and analysis of tumor response in mobile spine and sacral chordoma treated with definitive high-dose photon/proton radiation therapy. Int J Radiat Oncol Biol Phys. 2017;97(2): 254-262.

20. Park L, Delaney TF, Liebsch NJ, et al. Sacral chordomas: Impact of high-dose proton/photon-beam radiation therapy combined with or without surgery for primary versus recurrent tumor. Int J Radiat Oncol Biol Phys. 2006;65(5): 1514-1521.

21. Bostel T, Mattke M, Nicolay NH, et al. High-dose carbon-ion based radiotherapy of primary and recurrent sacrococcygeal chordomas: long-term clinical results of a single particle therapy center. Radiat Oncol. 2020;15(1):206.

22. Cote GM, Barysauskas CM, DeLaney TF, et al. A phase 1 study of nilotinib plus radiation in high-risk chordoma. Int $J$ Radiat Oncol Biol Phys. 2018;102(5):1496-1504.

23. Demizu Y, Imai R, Kiyohara H, et al. Carbon ion radiotherapy for sacral chordoma: a retrospective nationwide multicentre study in Japan. Radiother Oncol. 2021;154:1-5.

24. Imai R, Kamada T, Tsuji H, et al. Carbon ion radiotherapy for unresectable sacral chordomas. Clin Cancer Res. 2004; 10(17):5741-5746.

25. Imai R, Kamada T, Tsuji H, et al. Effect of carbon ion radiotherapy for sacral chordoma: results of Phase I-II and Phase II clinical trials. Int J Radiat Oncol Biol Phys. 2010;77(5): 1470-1476.

26. Imai R, Kamada $\mathrm{T}$, Sugahara $\mathrm{S}$, et al. Carbon ion radiotherapy for sacral chordoma. Br J Radiol. 2011;84(special issue 1): S48-S54.

27. Imai R, Kamada T, Araki N. Carbon ion radiation therapy for unresectable sacral chordoma: an analysis of 188 cases. Int $J$ Radiat Oncol Biol Phys. 2016;95(1):322-327.

28. Matsumoto K, Imai R, Kamada T, et al. Impact of carbon ion radiotherapy for primary spinal sarcoma. Cancer. 2013; 119(19):3496-3503.

29. Nishida Y, Kamada T, Imai R, et al. Clinical outcome of sacral chordoma with carbon ion radiotherapy compared with surgery. Int J Radiat Oncol Biol Phys. 2011;79(1):110-116.

30. Preda L, Stoppa D, Fiore MR, et al. MRI evaluation of sacral chordoma treated with carbon ion radiotherapy alone. Radiother Oncol. 2018;128(2):203-208.

31. Schulz-Ertner D, Nikoghosyan A, Thilmann C, et al. Results of carbon ion radiotherapy in 152 patients. Int J Radiat Oncol Biol Phys. 2004;58(2):631-640.

32. Serizawa I, Imai R, Kamada T, et al. Changes in tumor volume of sacral chordoma after carbon ion radiotherapy. $J$ Comput Assist Tomogr. 2009;33(5):795-798.

33. Demizu Y, Mizumoto M, Onoe T, et al. Proton beam therapy for bone sarcomas of the skull base and spine: a retrospec- 
tive nationwide multicenter study in Japan. Cancer Sci. 2017; 108(5):972-977.

34. Uhl M, Welzel T, Jensen A, et al. Carbon ion beam treatment in patients with primary and recurrent sacrococcygeal chordoma. Strahlenther Onkol. 2015;191(7):597-603.

35. Wu S, Li P, Cai X, et al. Carbon ion radiotherapy for patients with extracranial chordoma or chondrosarcoma-initial experience from Shanghai Proton and Heavy Ion Center. $J$ Cancer. 2019;10(15):3315-3322.

36. Zhang H, Yoshikawa K, Tamura K, et al. Carbon-11-methionine positron emission tomography imaging of chordoma. Skeletal Radiol. 2004;33(9):524-530.

37. Mima M, Demizu Y, Jin D, et al. Particle therapy using carbon ions or protons as a definitive therapy for patients with primary sacral chordoma. Br J Radiol. 2014;87(1033): 20130512

38. Schoenthaler R, Castro JR, Petti PL, et al. Charged particle irradiation of sacral chordomas. Int J Radiat Oncol Biol Phys. 1993;26(2):291-298.

39. Tsugawa D, Komatsu S, Demizu Y, et al. Space-making particle therapy with surgical spacer placement in patients with sacral chordoma. J Am Coll Surg. 2020;230(2):207-215.

40. Indelicato DJ, Rotondo RL, Begosh-Mayne D, et al. A prospective outcomes study of proton therapy for chordomas and chondrosarcomas of the spine. Int J Radiat Oncol Biol Phys. 2016;95(1):297-303.

41. Murray FR, Snider JW, Schneider RA, et al. Prognostic factors for spinal chordomas and chondrosarcomas treated with postoperative pencil-beam scanning proton therapy: a large, single-institution experience. J Neurosurg Spine. 2020;32(6): 921-930.

42. Snider JW, Schneider RA, Poelma-Tap D, et al. Long-term outcomes and prognostic factors after pencil-beam scanning proton radiation therapy for spinal chordomas: a large, singleinstitution cohort. Int J Radiat Oncol Biol Phys. 2018;101(1): 226-233.

43. Staab A, Rutz HP, Ares C, et al. Spot-scanning-based proton therapy for extracranial chordoma. Int J Radiat Oncol Biol Phys. 2011;81(4):e489-e496.

44. Tran S, Puric E, Walser M, et al. Early results and volumetric analysis after spot-scanning proton therapy with concomitant hyperthermia in large inoperable sacral chordomas. $\mathrm{Br} J \mathrm{Ra}$ diol. 2020;93(1107):20180883.

45. Austin JP, Urie MM, Cardenosa G, Munzenrider JE. Probable causes of recurrence in patients with chordoma and chondrosarcoma of the base of skull and cervical spine. Int $J$ Radiat Oncol Biol Phys. 1993;25(3):439-444.

46. North American Spine Society. Levels of evidence for primary research question as adopted by the North American Spine Society January 2005. Accessed March 22, 2021. https://www.spine.org/Portals/0/assets/downloads/Research ClinicalCare/LevelsOfEvidence.pdf

47. Matsumoto T, Imagama S, Ito Z, et al. Total spondylectomy following carbon ion radiotherapy to treat chordoma of the mobile spine. Bone Joint J. 2013;95-B(10):1392-1395.

48. Akiyama T, Ogura K, Gokita T, et al. Analysis of the infiltrative features of chordoma: the relationship between microskip metastasis and postoperative outcomes. Ann Surg Oncol. 2018;25(4):912-919.

49. Zuckerman SL, Bilsky MH, Laufer I. Chordomas of the skull base, mobile spine, and sacrum: an epidemiologic investigation of presentation, treatment, and survival. World Neurosurg. 2018;113:e618-e627.

50. Charest-Morin R, Dirks MS, Patel S, et al. Ewing sarcoma of the spine: prognostic variables for survival and local control in surgically treated patients. Spine (Phila Pa 1976). 2018; 43(9):622-629.

51. Dea N, Fisher CG, Reynolds JJ, et al. Current treatment strategy for newly diagnosed chordoma of the mobile spine and sacrum: results of an international survey. J Neurosurg Spine. 2018;30(1):119-125.

52. Kirkpatrick JP, van der Kogel AJ, Schultheiss TE. Radiation dose-volume effects in the spinal cord. Int J Radiat Oncol Biol Phys. 2010;76(3)(suppl):S42-S49.

53. Kumar N, Madhu S, Bohra H, et al. Is there an optimal timing between radiotherapy and surgery to reduce wound complications in metastatic spine disease? A systematic review. Eur Spine J. 2020;29(12):3080-3115.

54. Newhauser WD, Zhang R. The physics of proton therapy. Phys Med Biol. 2015;60(8):R155-R209.

55. Houdek MT, Rose PS, Hevesi M, et al. Low dose radiotherapy is associated with local complications but not disease control in sacral chordoma. J Surg Oncol. 2019;119(7):856-863.

56. Jin CJ, Berry-Candelario J, Reiner AS, et al. Long-term outcomes of high-dose single-fraction radiosurgery for chordomas of the spine and sacrum. J Neurosurg Spine. 2019;32(1): 79-88.

57. van Wulfften Palthe O, Jee KW, Bramer JAM, et al. What is the effect of high-dose radiation on bone in patients with sacral chordoma? A CT study. Clin Orthop Relat Res. 2018; 476(3):520-528.

58. Boriani S, Pipola V, Cecchinato R, et al. Composite PEEK/ carbon fiber rods in the treatment for bone tumors of the cervical spine: a case series. Eur Spine J. 2020;29(12):32293236.

59. Tedesco G, Gasbarrini A, Bandiera S, et al. Composite PEEK/carbon fiber implants can increase the effectiveness of radiotherapy in the management of spine tumors. $J$ Spine Surg. 2017;3(3):323-329.

60. Mastella E, Molinelli S, Magro G, et al. Dosimetric characterization of carbon fiber stabilization devices for post-operative particle therapy. Phys Med. 2017;44:18-25.

61. Müller BS, Ryang YM, Oechsner M, et al. The dosimetric impact of stabilizing spinal implants in radiotherapy treatment planning with protons and photons: standard titanium alloy vs. radiolucent carbon-fiber-reinforced PEEK systems. $J$ Appl Clin Med Phys. 2020;21(8):6-14.

62. Kurtz SM, Devine JN. PEEK biomaterials in trauma, orthopedic, and spinal implants. Biomaterials. 2007;28(32): 4845-4869.

63. Adler D, Akbar M, Spicher A, et al. Biomechanical study of a novel, expandable, non-metallic and radiolucent $\mathrm{CF} / \mathrm{PEEK}$ vertebral body replacement (VBR). Materials (Basel). 2019; 12(17):2732.

64. Varga PP, Szövérfi Z, Fisher CG, et al. Surgical treatment of sacral chordoma: prognostic variables for local recurrence and overall survival. Eur Spine J. 2015;24(5):1092-1101.

65. Fisher CG, Versteeg AL, Dea N, et al. Surgical management of spinal chondrosarcomas. Spine (Phila Pa 1976). 2016; 41(8):678-685.

66. Arshi A, Sharim J, Park DY, et al. Chondrosarcoma of the osseous spine: an analysis of epidemiology, patient outcomes, and prognostic factors using the SEER registry from 1973 to 2012. Spine (Phila Pa 1976). 2017;42(9):644-652.

67. Arshi A, Sharim J, Park DY, et al. Prognostic determinants and treatment outcomes analysis of osteosarcoma and Ewing sarcoma of the spine. Spine J. 2017;17(5):645-655.

68. Terlizzi M, Le Pechoux C, Salas S, et al. Postoperative radiation therapy in patients with extracranial chondrosarcoma: a joint study of the French Sarcoma Group and Rare Cancer Network. Int J Radiat Oncol Biol Phys. 2020;107(4):726-735.

\section{Disclosures}

Dr. Goodwin has received grants from the Robert Wood Johnson Harold Amos Medical Faculty Development Program and the Burroughs Wellcome Fund and received the NIH/NINDS K12 NRCDP Physician Scientist Award. Dr. Schwab is a consultant 
for DePuy Synthes and Stryker. Dr. Sciubba is a consultant for Baxter, DePuy Synthes, Medtronic, and Stryker.

\section{Author Contributions}

Conception and design: Pennington. Acquisition of data: Pennington, Ehresman. Analysis and interpretation of data: Pennington, Ehresman. Drafting the article: Pennington,

Ehresman. Critically revising the article: all authors. Reviewed submitted version of manuscript: all authors. Approved the final version of the manuscript on behalf of all authors: Sciubba.

\section{Correspondence}

Daniel M. Sciubba: Zucker School of Medicine at Hofstra, Long Island Jewish Medical Center and North Shore University Hospital, Northwell Health, Manhasset, NY. dsciubba1@northwell.edu. 